\title{
Éducation et conscience historique
}

\section{Horio Teruhisa}

Traducteur : Christophe Sabouret

\section{CpenEdition}

\section{Journals}

Édition électronique

URL : http://journals.openedition.org/ries/3254

DOI : 10.4000/ries.3254

ISSN : 2261-4265

Éditeur

Centre international d'études pédagogiques

Édition imprimée

Date de publication : 1 mars 1997

Pagination : 107-115

ISSN : 1254-4590

\section{Référence électronique}

Horio Teruhisa, «Éducation et conscience historique », Revue internationale d'éducation de Sèvres [En ligne], 13 | 1997, mis en ligne le 24 juillet 2013, consulté le 26 avril 2019. URL : http://

journals.openedition.org/ries/3254; DOI : 10.4000/ries.3254 


\section{Éducation \\ et conscience \\ historique ${ }^{1}$}

Horio Teruhisa ${ }^{2}$

S'interroger sur ce qu'est le contemporain revient en fait à s'interroger sur ce qu'est, pour chacun d'entre nous, vivre en tant que contemporain. Mais, s'il se présente souvent sous la forme de la confusion, le contemporain offre aussi la possibilité, à travers la confusion, de fonder un espoir. Si je pose ce postulat, c'est parce que j'ai en mémoire les propos célèbres du Président de l'ex-RFA, Weizsäcker, qui dit, en substance, que celui qui ferme les yeux sur le passé est finalement aveugle sur le présent et que notre devoir est de graver le passé dans nos cœurs afin d'avancer. En effet, il est important de graver le passé dans nos cœurs. Mais en même temps, avancer ou, plus simplement, vivre aujourd'hui, n'est possible qu'à la condition de parvenir aussi à déchiffrer l'avenir dans ce contemporain confus. Parce qu'elle peut nous permettre de fonder un choix de valeurs en vue de l'avenir, cette orientation est importante. Enfin, limiter les propos de Weizsäcker au seul devoir de graver le passé dans nos cœurs peut présenter le danger d'encourir l'accusation, dont il sera question plus loin, de proposer une vision autodépréciative de l'histoire (Jigyaku-shikan). J'examinerai ici si cette insistance sur l'avenir et, en particulier sur la question de la réponse à l'avenir et du choix des valeurs en vue de l'avenir, n'est pas ce qui différencie le plus les historiens des personnes qui ont affaire à l'éducation.

\section{Enjeux}

Quoi qu'il en soit, l'interrogation sur le contemporain peut se ramener aux espoirs que l'on peut légitimement fonder dans l'éducation. Or, la recherche actuelle en sciences de l'éducation donne à penser qu'elle a perdu toute capacité à se prononcer sur l'avenir. Ceci est manifeste en particulier chez les jeunes chercheurs, qui, à la suite de Foucault et dans le contexte du débat autour de la post-modernité, soulignent le caractère oppressif de l'école en tant qu'institution ou, à la suite d'Althusser, ne perçoivent l'école ou l'éducation que comme un appareil idéologique d'État. Surmonter cette défiance, passe peut-être par la mise en perspective de l'histoire de ces cinquante dernières années et, en particulier, de 1945. Parce qu'elle marque aussi bien dans l'évolution de l'histoire

1 Discours prononcé au Conseil national de la recherche scientifique du Japon (Nihon Gakujutsu Kaigı), le 19 octobre 1996. Titre en japonais : Kyôiku to Rekishi-ishiki.

Ce texte a été traduit et annoté par Christophe Sabouret, sauf mention contraire.

2 En Japonais le nom précède le prénom, cette règle a été respectée dans tout le texte (N.D.L.R.). 
moderne du Japon que dans l'histoire du monde un point d'évolution déterminant, l'année 1945 revêt une importance particulière. En outre, au regard de l'histoire de l'après-guerre, la date de 1945 nous commande de réfléchir à sa signification. Si cette démarche me semble importante aujourd'hui, c'est parce que 1945 marque tout d'abord l'entrée dans une histoire planétaire et, s'agissant du Japon et de l'évolution de l'histoire moderne du Japon, 1945 marque le passage de la Constitution impériale (1889) à la Constitution actuelle (1946) en même temps que le passage du rescrit impérial sur l'éducation (1890) à la loi fondamentale de l'éducation (1947).

A mon avis, le contemporain commence en 1945 et fait suite à une période moderne classique et à une période moderne postérieure dont les années 1870 marquent le début. Ce qui précède n'est pas sans rapport avec ma propre histoire qui se confond avec celle de l'avant-guerre à celle de l'aprèsguerre en passant par celle de la guerre. Et, si l'on songe à ce que dit Ôe Kenzaburô ${ }^{3}$ lorsqu'il parle de s'en tenir à la démocratie de l'après-guerre, la question est de savoir comment, à travers un vécu, transmettre une expérience aux générations à venir. Enseignant moi-même, je dois reconnaître que j'éprouve les plus grandes difficultés à réaliser ce vœu. J'ouvre ici une parenthèse qui fera peut-être mieux comprendre ce que je veux dire. Mon père est mort à la guerre, aussi suis-je ce qu'on appelle un descendant d'une "âme héroïque » (Eirei). C'est pourquoi j'ai suivi, non sans inquiétudes, le débat sur 108 la Déclaration de la Diète au sujet de la guerre (juin 1995). Je dois cependant dire mon indignation devant l'affirmation selon laquelle critiquer la guerre de Quinze Ans (1931-1945 : de l'incident du Pont Marco-Polo à la défaite en 1945, en passant par la seconde guerre sino-japonaise, 1931-1945, et la guerre du Pacifique, 1941-1945) revient en fait à insulter la mémoire des morts pour la patrie. En tant que membre de l'Association pacifiste des parents de défunts, ce genre d'affirmation me choque. C'est dire toute l'importance de sa propre histoire.

Dans cette perspective, il me revient en mémoire ce que mon professeur Maruyama Masao, décédé en août dernier, a dit au moment des débats au sujet du renouvellement du traité de sécurité nippo-américain en 1960. Il soulignait alors la nécessité de ne pas perdre de vue l'essentiel ou la matrice des choses. Autrement dit, ne nous enjoignait-il pas de remonter jusqu'à cette matrice de l'après-guerre et, plus précisément, jusqu'au 15 août $1945^{4}$ ? Dire cela, c'est dire combien il nous importe de préserver en nous l'esprit qui nous a animé au lendemain de la défaite et qui nous a permis de construire, aux

3 Prix Nobel de littérature en 1995, professeur au département de français de l'université de Tokyo (N.D.L.R.).

4 A cette date, l'empereur fit diffuser, par radio, le " rescrit mettant fin à la guerre " qu'il avait lui-même lu et fait enregistrer (N.D.L.R.). 
milieux des ruines, un nouveau Japon. Certains verront ici peut-être une trop grande liberté dans l'interprétation des propos de Maruyama. Mais, mon axiomatique n'ayant jamais été très éloignée de ses propositions, je ne crois pas trop trahir sa pensée. C'est pourquoi j'attache une si grande importance au 15 août qui inaugure aussi bien pour le monde que pour le Japon une nouvelle ère : l'ère de l'histoire planétaire. Si parler de paradigme a un sens, alors 1945 marque à l'évidence un changement de paradigme.

\section{Histoire critique ou " autodépréciation " ?}

Mais qu'en est-il aujourd'hui, cinquante ans après, de ce paradigme? $\mathrm{Au}$ vrai, la situation actuelle ne porte guère à l'optimisme. On assiste tout d'abord à la troisième offensive sur les manuels scolaires depuis 1945 (1955, $1982,1995)^{5}$. D'un côté, il est vrai qu'il existe au Japon un fort courant qui conçoit qu'on admette une responsabilité de la guerre, visible notamment dans la reconnaissance par l'ex-Premier ministre Hosokawa Morihiro du caractère agressif des actes de guerre commis par le Japon et dans la reconnaissance, malgré tout, d'une responsabilité de nature morale par l'actuel Premier ministre Hashimoto Ryûtarô au sujet de la question des "épouses de consolation " (Ianfu) affectées au service du «repos» du soldat japonais pendant la guerre. D'un autre côté, on constate toute une série de manifestations qui, autour des "faucons ", notamment Okano Seiryô, visent à contrecarrer le courant dont il vient d'être question. Dans les années 1994-1995, il y eut les «sorties » et autres déclarations intempestives sur la guerre du ministre de la Justice Nagai et du secrétaire à l'Environnement Sakurai. Il y eut ces associations, composées ou représentées par des conservateurs, tel que Okano Seiryô qui présida la très droitière Fédération des parlementaires de la Diète, qui militèrent en faveur d'une histoire, selon elles, plus "correcte » et qui contribuèrent à infléchir dans leur sens le contenu de la Déclaration de la Diète au sujet de la guerre. Il s'ensuit que le sens et la portée de la guerre sont aujourd'hui amoindris, voire niés, à tout le moins relativisés.

Que pensent nos voisins asiatiques de tout cela ? Professeur à l'université d'Okinawa, Go Song Min nous en donne une première indication. Dans le quotidien Asahi Shinbun du 7 juin 1996, il écrit: "C'est l'année dernière, cinquante après la fin de la guerre, à l'occasion de la Déclaration de la Diète au sujet de la guerre, que la Chine a pu ouvrir les yeux sur sa perception du Japon. Aboutissement de nombreuses difficultés, cette déclaration est un texte curieux où l'on apprend surtout que le Japon n'est pas seul en cause, que les torts sont

5 Sur ce problème des manuels et leur système de contrôle, il est particulièrement intéressant de lire : Horio Teruhisa, L'éducation au Japon, Paris, CNRS sociologie, 1993, p.15, 109, 116-117, 120-121, 128-140 (N.D.L.R.). 
partagés et où l'on cherche en vain la mention d'excuses ou de regrets. Ceci ne peut que plonger les Asiatiques dans la consternation. En fait, l'absence d'excuses ou de regrets traduit le sentiment réel du Japon (...). » Dans ces conditions, il n'est peut-être pas surprenant que le débat porte aujourd'hui sur la question de la confiance à accorder au Japon.

Mais, si elles nous sont largement connues à l'échelon politique, on sait moins que les tentatives de liquidation du passé connaissent aujourd'hui un regain de vigueur au sein même de l'enseignement. Elles se manifestent sous la forme de la critique qui s'en prend à l'éducation qui, depuis l'après-guerre, trouve son fondement dans la paix ou à l'enseignement de l'histoire qui repose sur la reconnaissance de la guerre de Quinze Ans et du caractère agressif de celle-ci, identifiée abusivement à une vision autodépréciative de l'histoire. Or, il se trouve que l'un des porte-parole de cette critique n'est autre que Fujioka Nobukatsu ${ }^{6}$. Dirigée initialement contre la prétendue vision de l'histoire fondée sur le procès de Tokyo ${ }^{7}$, puis contre le Komintern, enfin contre la vision de l'histoire qu'il considère comme autodépréciative, la critique de Fujioka est aujourd'hui très appréciée par des gens comme Okano Seiryô. Tout se passe comme s'il se trouvait enfin un représentant du champ intellectuel pour exprimer tout haut ce qu'ils pensaient tout bas. Le cas est pendable et me fait irrésistiblement penser à ce que Benda disait de la trahison des clercs.

Parmi les propositions de Fujioka, n'en citons qu'une, celle qui le 110 conduit à considérer la question des "épouses de consolation » comme une atteinte grave à la fierté des Japonais, en même temps qu'à lancer un appel en faveur d'un mouvement qui réclamerait la suppression dans les manuels scolaires de toutes mention de ces faits. Invité à " plancher» devant le groupe de réflexion politique du parti libéral démocrate le 5 septembre dernier, le même Fujioka a réitéré sa critique et dénoncé le scandale que représentait à ses yeux la mention de ces faits dans les manuels scolaires, faits - toujours selon lui sans fondements historiques, qui ne viseraient en fait qu'à affaiblir moralement les Japonais et à interdire qu'ils ne s'identifient au fait d'être Japonais. Dans un entretien accordé à une revue, Fujioka dit encore, en substance, que, entrées par effraction dans les manuels scolaires du temps de la cohabitation entre le parti libéral démocrate et le parti socialiste, les «épouses de consolation » devraient connaître l'épreuve de la gomme qui, d'un geste, les bifferait. On est donc face à un personnage qui d'un côté propose de biffer dans les manuels scolaires les passages de l'histoire qu'il juge indésirables et qui de l'autre appelle à la mobilisation afin de promouvoir ses idées. Cette situation n'est que trop regrettable.

6 Un de mes anciens collègues, actuellement en poste à l'université de Tokyo.

7 Ce procès, qui s'est déroulé de mai 1946 à novembre 1948, concernait les criminels de guerre et a abouti à l'éxécution de certains d'entre eux (N.D.L.R.). 
Comment en est-on arrivé là ? Fujioka nous apprend qu'il a fait un séjour d'étude aux États-Unis aussitôt après la guerre du Golfe et que c'est à cette occasion que la lumière s'est fait jour en lui. L'heureux homme ! D'une part, la guerre du Golfe lui a fait prendre conscience de l'existence de guerres justes ; d'autre part, la lecture de La Justice du Vainqueur (Victor's Justice, 1971) de Richard H. Minear l'a amené à reconsidérer, de façon critique cette fois-ci, le procès de Tokyo. Connaissant moi-même personnellement Minear, je doute fort qu'il abonde dans le sens de Fujioka. En outre, ayant lu attentivement le livre de Minear, je n'y ai pas lu autre chose que la condamnation d'une justice unilatérale en même temps que le refus, sous-tendu par la condamnation qui précède, de cautionner les crimes commis alors par les États-Unis au Vietnam. Extraire de son contexte le livre de Minear, c'est non seulement rater l'essentiel de la thèse qu'il développe mais encore oublier son souci de paix en même temps que les fort belles traductions de recueils sur Hiroshima et la bombe atomique que cet historien a faites. A l'évidence, Minear qui est invoqué par Fujioka, ne poursuivait ni ne poursuit les même desseins. Lors des premières déclarations de Fujioka, j'étais partisan de l'indifférence. Mais à mon grand regret, les choses prennent une tournure décidément trop politique pour que l'on puisse continuer à affecter le détachement.

\section{Nouveaux objectifs}

Dans ces conditions, que faire ? Quel projet opposer à ces tentatives de liquidation du passé impérialiste du Japon? Si la guerre avait été simplement le combat de la démocratie contre le fascisme, de la démocratie contre le militarisme, les choses seraient simples. En tout cas, elles seraient suffisamment simples pour faire apparaître sans fondement la thèse d'une vision autodépréciative de l'histoire. Mais les choses ne sont pas aussi simples. De sorte que, faute de proposer une histoire qui échappe aux oppositions simples en même temps qu'à la mise en cause de la seule culpabilité du Japon, on risque de donner des arguments à la thèse de cette vision discréditée de l'histoire. Fujioka ne s'est d'ailleurs pas privé de dénoncer comme vision autodépréciative de l'histoire les travaux de l'Association pour l'enseignement de l'histoire, notamment ceux de Honda Kôei. Il est à signaler cependant, qu'au sein de cette même Association, une réflexion s'est engagée depuis afin de reconsidérer l'enseignement de l'histoire.

Parallèlement, le groupe de recherche sur l'histoire comparative poursuit ses activités. Il y a eu un séminaire sur l'enseignement de l'histoire en Corée et au Japon (1991). A cette occasion, l'intervention de Mera Seijirô, professeur au collège de Kaisei, m'a particulièrement marqué. Faisant le bilan de son enseignement, Mera a souligné la nécessité de dépasser un enseignement fondé sur la dénonciation, dont il ne méconnaît pas l'intérêt et de promouvoir une histoire qui prenne en compte aussi la question de la fierté. M'intéressant moi-même depuis quelques temps à la mise en perspective internationale de l'enseigne- 
ment, l'intervention de Mera rejoint certaines de mes préoccupations. Dans cette perspective, je pense aussi que l'enseignement de l'histoire ne peut pas se limiter à la dénonciation de la responsabilité relative à la guerre de Quinze Ans mais peut, et donc doit, intégrer la problématique de la fierté. Cette problématique de la fierté n'est pas étrangère à la question du patriotisme, aussi est-il nécessaire d'en passer par l'histoire; autrement dit par le choix attentif et raisonné des faits qui pourraient la servir. Par exemple, il y aurait un profit à rappeler, à travers les figures de Nakae Chômin, Kôtoku Shûsui, Uchimura Kanzô, Yanaihara Tadao, Ishibashi Tanzan, Okakura Tenshin, Yanagi Muneyoshi, etc., la tradition pacifiste de l'avant-guerre. Pour l'éducation, le profit serait également appréciable, si l'on évoquait, à travers le Mouvement pour une nouvelle éducation ${ }^{8}$, l'existence d'une éducation de résistance qui s'est opposée à la guerre de Quinze Ans. Ce faisant, les élèves apprendraient qu'il n'y avait pas seulement des envahisseurs parmi leurs aînés.

\section{Débat sur l'article 9}

Tournons-nous à présent vers une autre cible de la critique: la Constitution et, en particulier, l'article $9{ }^{9}$. Ces temps-ci, on entend dire que ce pacifisme constitutionnel est limité à un seul pays, le Japon en l'occurrence. Certains estiment qu'il date d'un autre âge. Ou encore, Nagao Ryûichi, spécialiste de la philosophie du droit, qui s'exprime dans la revue This is Yomiuri (novembre 1996), déclare en substance que, né de la conception militaire tout à fait particulière de Mac-Arthur, l'article 9 est le fruit d'un mélange entre droit et religion, et de la théorie, soutenue par les pays alliés, de l'affaiblissement du Japon. Ainsi compris, l'article 9 peut devenir l'objet du débat qui envisage sa révision. Pourtant, dans sa grande majorité, la communauté des juristes s'accorde pour soutenir la thèse de l'intervention commune des États-Unis et du Japon dans l'élaboration de l'article 9. Quant à moi, j'insiste plus particulièrement sur l'initiative de Shidehara Kijûrô et, en particulier, sur l'entrevue qu'il a eue le 24 janvier 1946 avec Mac-Arthur. Selon le propre témoignage de MacArthur, le $1^{\text {er }}$ mai 1951, devant le Sénat américain, il semble que ce soit Shidehara qui ait, le premier, évoqué cette clause du pacifisme constitutionnel.

Mais l'essentiel du débat ne se limite pas à l'initiative de Shidehara en faveur de l'article 9. Il porte également sur la portée, à travers les cinquante dernières années et le monde, de l'article 9. Cet article n'importe pas seulement

8 Mouvement du début des années trente.

9 Texte de l'article 9: "Aspirant sincèrement à une paix internationale basée sur l'ordre et la justice, le peuple japonais renonce pour toujours à la guerre (...) comme moyen de régler les conflits internationaux. Afin d'atteindre le but défini par le paragraphe précédent, les forces de terre, de mer et de l'air (...) ne seront jamais maintenues. " 
aux Japonais mais, un certain nombre de prises de positions en témoignent, à des étrangers aussi. Je pense notamment à C. Overby, à B. Gorden, ou encore à Liu Pei Jing qui, considérant cet article 9 comme une forme d'engagement international pris face aux vingt millions de morts asiatiques occasionnés par la guerre, regretterait qu'on en change, aussi longtemps qu'il restera un seul asiatique pour ne pas le souhaiter. Né de l'examen de conscience, après la défaite, face au militarisme et aux guerres d'agression, l'article 9 est aussi, si l'on songe au nucléaire et à l'histoire planétaire, extrêmement précieux. C'est pourquoi, il me semble si important de penser le Japon, non pas pour lui-même, mais dans et par rapport à l'Asie.

\section{Le Japon et les pays d'Asie}

Dans cette perspective, le petit livre de Liu Pei Jing, Connaissons mieux l'Asie (Motto shirô Asia), ouvre la voie à une réflexion sur la façon de rendre plus actuel l'enseignement de l'histoire au Japon. C'est peut-être en cela que l'enseignement de l'histoire se distingue de la recherche en histoire. Quoi qu'il en soit, s'il est malaisé de répondre à la question, peut-être peut-on proposer quelques pistes. Par exemple, comment enseigner l'assassinat, qui précède l'annexion de la Corée par le Japon en 1910, de Itô Hirobumi, le résident japonais en Corée, par Ahn Jung Gun (oct. 1909) ? Le sujet est délicat et les différences d'appréciations au Japon et en Corée notables. Considéré comme un terroriste par le Japon, Ahn Jung Gun est traité comme un patriote en Corée. Dès lors, comment présenter Ahn Jung Gun aux enfants japonais ? Ne présenter que le point de vue de la Corée risque fort de ne pas emporter leur adhésion. La question paraît insoluble. Or, il se trouve que, alors membre de la police militaire et âgé de 24 ans, Chiba Tohichi, qui a surveillé Ahn Jung Gun après son incarcération, a recueilli ses dernières volontés sous la forme d'un bref message écrit. Dans le temple de Dairinji, en Corée, un même monument est dédié depuis les années soixante-dix à la mémoire de Ahn Jung Gun et de Chiba Tohichi. N'est-ce pas là l'une des expressions possibles de notre aspiration à la paix partagée? N'est-ce pas là en tout cas, à travers l'enseignement de ce récit, l'occasion d'intéresser à autrui les enfants? D'autres récits de nature similaire nous sont aujourd'hui offerts.

Nous connaissons tous l'histoire de Son Gyon, ce marathonien coréen, vainqueur aux jeux olympiques de Berlin de 1936, qui a vu disparaître de la photographie prise lors de la cérémonie de remise des médailles, le Hinomaru, le drapeau du Japon, qui ornait son survêtement. L'incident entraîna la condamnation du journal coréen Tôa Nippô par le gouverneur général japonais de la Corée. Or, il se trouve que le porteur de la flamme olympique lors des jeux de Séoul (1988) fut précisément Son Gyon. Le rapprochement de ces deux faits offre la possibilité, me semble-t-il, d'intéresser les enfants à l'histoire, en les amenant tout d'abord à s'interroger sur les raisons du choix de ce septatuagé- 
naire pour incarner, dans les années quatre-vingt, les valeurs de l'olympisme, puis sur la période de l'avant-guerre. Dans cet esprit, la lecture du petit livre de Yamamoto Tenjin, Enseigner l'affaire de la disparition du Hinomaru (Hinomarumasshô-jiken o jugyô suru), est très stimulante. Elle nous apprend, pratiquement, puisque Yamomoto s'est livré à cet exercice aussi bien au Japon qu'en Corée, qu'il ne suffit pas d'enseigner l'histoire de la période coloniale pour intéresser les enfants à l'histoire.

Il est d'autres faits, comme la déclaration d'indépendance de la Corée, le $1^{\text {er }}$ mars 1919, et la position face à elle d'un Japonais comme Yanagi Muneyoshi, qui sont susceptibles de faire l'objet d'un enseignement de l'histoire plus actuel. Le rappel des lettres de protestation que des artistes envoyèrent après que le Japon ait fait détruire la porte principale du palais royal à Séoul, pour y construire le siège du gouvernement général de la Corée, est propre à faciliter une approche plus humaine de l'histoire. Il est également possible de tirer parti du livre, rédigé en anglais, de Okakura Tenshin, Le livre du thé (Cha no Hon), pour approfondir la réflexion sur la paix. Dans ce livre, l'auteur nous dit qu'il est encore préférable de ne pas verser le sang, quitte à être ainsi considéré comme un pays non civilisé, puisque ce n'est qu'après avoir perpétré des massacres (lors de la guerre russo-japonaise, 1904-1905) que le Japon, jusqu'alors considéré comme un pays arriéré, empêtré dans un art pacifique, a accédé au statut de pays civilisé aux yeux des occidentaux.

Ce qui précède ne définit en aucune manière un programme mais des pistes qui gagneraient, me semble-t-il, à être explorées afin de nuancer le point de vue qui consiste à mettre l'accent sur les guerres d'agression. Il reste que cet effort doit être mené dans le plus grand souci de l'exactitude historique. Il ne s'agit pas seulement d'inventer de belles histoires.

Le débat ne se limite donc pas ici à la seule question du niveau de l'enseignement mais s'étend à celle d'une forme de sensibilité, c'est-à-dire à celle du mode d'accès, à travers le passage dans l'ordre de la sensation, vers une forme de compassion. S'il est vrai que l'éducation relève de l'intelligence, autrement dit de la mise en relation, alors il est vrai aussi que cette intelligence ne relève pas seulement de l'ordre du savoir mais également de l'ordre de la sensation qui, sous-tendue par l'idée de la sympathie, rend possible les rapports humains. Saint-Exupéry ne nous a-t-il pas appris que «l'on ne voit bien qu'avec le cœur»? Enfin, qu'il me soit permis d'ajouter ici que rien ne me semble plus propre à faciliter l'accès à l'universel que de partir du concret ou, plus précisément, du particulier. Par exemple, la mise en évidence de l'universel qu'exprime le principe des droits de l'homme peut et doit participer de cette démarche. Il s'agit d'envisager l'universel à travers ses différences et ses spécificités. Par ailleurs, s'il est vrai que le passé le détermine, l'universel est aussi orienté vers 
l'avenir. L'histoire et l'enseignement de l'histoire gagneraient sûrement à intégrer cette dimension-là. Ce faisant, la connaissance pourrait contribuer à affermir notre pouvoir. On a coutume de dire que le savoir c'est le pouvoir. Mais la question serait plutôt de savoir comment faire en sorte que le pouvoir trouve son fondement dans le savoir. Comment rendre possible la démocratie qui reposerait sur le savoir de chacun au service de tous? 